Review paper

\title{
Review of skin dose calculation software in interventional cardiology
}

\author{
Françoise Malchair ${ }^{\mathrm{a}}$, Jérémie Dabin ${ }^{\mathrm{b}}$, Marine Deleu ${ }^{\mathrm{c}}$, Marta Sans Merce ${ }^{\mathrm{c}}$, Olivera Ciraj Bjelac ${ }^{\mathrm{d}}$, \\ Aoife Gallagher ${ }^{\mathrm{e}}$, Carlo Maccia ${ }^{\text {a, * }}$ \\ ${ }^{a}$ CAATS, 119 Grande Rue, 92310 Sèvres, France \\ ${ }^{\mathrm{b}}$ Belgian Nuclear Research Centre (SCK-CEN), Boeretang 200, BE-2400 Mol, Belgium \\ ${ }^{\mathrm{c}}$ University Hospitals of Geneva, Geneva, Switzerland \\ ${ }^{\mathrm{d}}$ University of Belgrade, Vinca Institute of Nuclear Sciences, P.O. Box 522, Belgrade, Serbia \\ ${ }^{\mathrm{e}}$ University Hospital Limerick, Ireland
}

\section{A R T I C L E I N F O}

\section{Keywords:}

Patient dosimetry

Interventional cardiology

Patient dose calculation software

Radiation protection

\begin{abstract}
A B S T R A C T
Purpose: In interventional cardiology, patients may be exposed to high doses to the skin resulting in skin burns following single or multiple procedures. Reviewing and analysing available software (online or offline) may help medical physicists assessing the maximum skin dose to the patient together with the dose distribution during (or after) these procedures.

Method and results: Capabilities and accuracy of available software were analysed through an extensive bibliography search and contacts with both vendor and authors. Their markedly differed among developers.

In total, 22 software were identified and reviewed according to their algorithms and their capabilities. Special attention was dedicated to their main features and limitations of interest for the intended clinical use.

While the accuracy of the 12 software products validated with measurements on phantoms was acceptable (within $\pm 25 \%$ ), the agreement was poor for the two products validated on patients (within $\pm 43 \%$ and $\pm 76 \%$, respectively). In addition, no software has been validated on angiographic units from all manufacturers, though several software developers claimed vendor-independent transportability. Only one software allows for multiple procedures dose calculation.

Conclusion: Large differences among vendors made it clear that work remains to be done before an accurate and reliable skin dose mapping is available for all patients.
\end{abstract}

\section{Introduction}

Since the $1980 \mathrm{~s}$, the introduction of more sophisticated endovascular devices in interventional cardiology (IC) allowed more complex procedures to be performed, thus potentially increasing the exposure to the patients, resulting in tissue reactions (e.g. skin burns) following single or multiple procedures.

As a consequence, reports on radiation-induced patient skin injuries started to emerge, highlighting the need for optimised and personalised patient dosimetry. Patient dosimetry, a necessity that has further strengthened, due to the increasing in the number of IC procedures over the years.

Assessing the Maximum Skin Dose (MSD) to the patient during (or after) those procedures together with 2D dose distribution is, as recommended by the International Commission on Radiation Protection (ICRP) Committee 3 [1], essential from patient radiation protection point of view.

Until recently, measurements using passive dosimeters were the only way to assess patient's MSD accurately. However, as these measurements are tedious to perform and expensive, they could not be

\footnotetext{
Abbreviations: AC, Attenuation Coefficient of table and mattress; BMI, Body-Mass Index; B, Backscatter Factor; CF, Calibration Factor; DICOM, Digital Imaging and

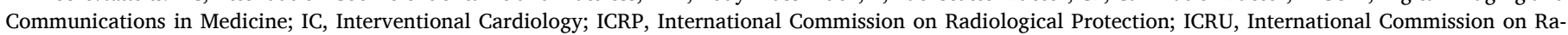

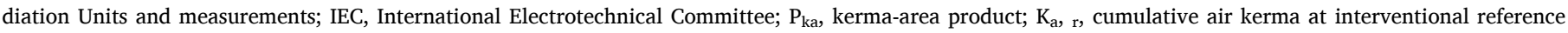

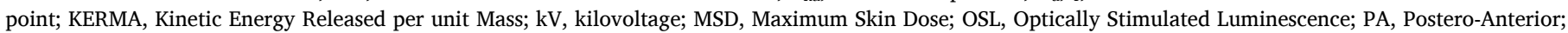

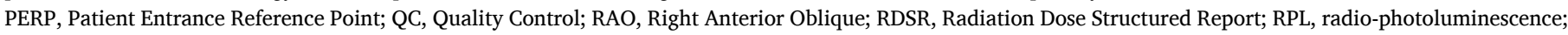
SDC, Skin Dose Calculation; TLD, Thermoluminescent Dosimeter.

* Corresponding author at: CAATS, 119 Grande Rue, 92310 Sèvres, France.

E-mail address: carlo.maccia@caats.fr (C. Maccia).
} 
performed routinely at clinics and a more convenient means to estimate MSD was needed [2].

Automated online and offline Skin Dose Calculation (SDC) solutions have therefore been developed. Online software use live data streaming from the angiographic systems to calculate skin doses directly during the procedures, while offline solutions use data stored in the Radiation Dose Structured Report (RDSR) to compute skin exposure post-procedure (see Table 1).

Currently, most vendors have implemented some form of skin dose calculations in their angiographic systems: from simple solutions only computing MSD values, to more advanced software solutions calculating a $2 \mathrm{D}$ or $3 \mathrm{D}$ distribution of the skin dose.

Offline SDC software solutions have also been developed in researchbased institutions or for commercial purposes, both as stand-alone products or integrated into the Dose Archiving and Communication Systems (DACS).

Within this context, the European project VERIDIC (Validation and Estimation of Radiation skIn Dose in Interventional Cardiology) was started in February 2018 [3].

One of the main objectives of the project was to review and validate existing SDC software products since medical physicists should be able to reliably assess patient dose in Interventional Cardiology while providing cath labs with dosimetry report in case of overexposed patients.

Large variety of dose software make such a task difficult and a critical evaluation of available products is essential from patient radiation protection point of view.

This paper focuses on the review of the software products.

\section{Material and methods}

Since significant dose differences can occur depending on whether a given parameter is accurately considered in the MSD calculation, available SDC software solutions were collected and analysed according to their calculation algorithms and their specific features $[4,5]$.

Physical parameters used for calculation of the MSD (tube voltage, filtration, beam orientation, table position, backscatter factor (B), table attenuation, air kerma-to-skin dose conversion coefficient) were also assessed.

A critical analysis of software was carried out by considering the

Table 1

List of available Maximum Skin Dose calculation software.

\begin{tabular}{lll}
\hline Software Name & Company name & References \\
Dose Map & GE healthcare & {$[7,8,9,10]$} \\
Dosewatch & GE healthcare & {$[11,12]$} \\
DTS & CANON Medical Systems & {$[13,14,15]$} \\
em.dose & ESPRIMED & {$[16,17]$} \\
Radimetrics & BAYER & NA \\
RDM & MEDSQUARE & {$[18]$} \\
DOSE & QAELUM & NA \\
UF-RIPSA & Non-commercial & {$[19,20,21]$} \\
MCAR & Non-commercial & {$[22,23]$} \\
FDEIR & Non-commercial & {$[24]$} \\
MCGPU & Non-commercial & {$[25]$} \\
TeamPlay & SIEMENS Healthineers & NA \\
Dosewise & PHILIPS & NA \\
Dosetrack & SECTRA & NA \\
Nexodose & BRACCO & {$[26,27]$} \\
Dose monitor & PACSHEALTH & NA \\
Dosem & INFINITT & NA \\
OpenSkin & Open Source & NA \\
PySkinDose & Open Source & {$[28]$} \\
SkinCare & Open Source & {$[29]$} \\
CAATSDOSE & CAATS & NA \\
DIDO & Hospital San Carlos Madrid Madrid & NA \\
\hline
\end{tabular}

MCAR: Mayo Clinic Arizona\&Rochester; CAATS:Centre d'Assurance de qualité des Applications Technologiques dans le domaine de la Santé; DTS: Dose Tracking Software; DIDO : Dicom Dose ; NA : Not Available. following properties:

- their calculation algorithms;

- the physical factors considered in the calculation of the MSD estimates;

- the modelling of patient's body shape;

- their capabilities of providing users with a 2D or 3D-dose distribution (graphical dose representations were qualitatively compared).

In order to gather all this information, the four major manufacturers of angiographic systems as well as all other developers included in Table 1 were contacted by mail. Some of them provided documents (not only published papers) and technical reports detailing the way the MSD were assessed and the modelling of the patient body shape. Technical information associated with SDC software developed by independent companies was also obtained through a literature review and/or personal contacts with the developers. It was not possible to obtain information about the calculation details for all considered systems. This was due to a lack of answers. Research laboratories were not contacted because sufficient data was provided in their published papers.

Both online and offline software (i.e. whether software provides the user with the MSD and possibly the dose distribution during or after the procedure, respectively) developed after 2000 were considered. Older software that was not available anymore like the program of den Boer et al. [5] was not considered. All products not using the RDSR as a source of information, thus missing the information about the fluoroscopy part of the procedures were also omitted.

\section{Results}

\subsection{Software products}

A total of 22 software products were identified. Most of them (18 out of 22) were "offline" systems. From a radiation protection point of view, "online" systems are preferable since they allow optimization of the MSD during the procedure.

Table 1 lists the software that were collected until the end of the VERIDIC project. The product name, manufacturer of the software if relevant and publication references describing the features of the software are listed in Table 1 . Where no reference was available, direct contact with the authors provided documentation about the software.

Among the listed MSD software, only 4 were online, namely Dose, MCGPU, DoseMap and DTS, out of 16 contacted software designers 13 provided an answer, while Philips, Siemens and SECTRA did not respond to the survey. Six non-commercial designers were not directly contacted since their publications were self-explanatory.

\subsection{Dose calculation}

The MSD calculation formula used by different manufacturers and non-commercial developers is presented below.

There is a clear agreement among the developers on how to assess the MSD value.

Except for MC-GPU and FDEIR, which model the particle transport via Monte Carlo (MC) simulations, all listed software use a formula comparable to the methodology proposed by Jones and Pasciak [30] for systems compliant with IEC standards.

The displayed air kerma at the reference point $\left(\mathrm{K}_{\mathrm{a}, \mathrm{r}}\right)$ [31] is corrected to account for the calibration of the $\mathrm{K}_{\mathrm{a}, \mathrm{r}}$ against quality control measurements, the table and mattress attenuation, the contribution of the backscattered radiation, the distance between the reference point and the actual patient entrance point and the conversion of air kerma to dose in tissue as follows:

Skindose $=K_{a, r} \times C F \times A C \times B \times\left(\frac{d_{\text {ref }}}{d_{\text {perp }}}\right)^{2} \times f_{\text {skin }}$ 
Where :

$C F$ is the calibration factor or the ratio between the measured and the displayed, $K_{a, r}$,

$A C$ is the attenuation coefficient of the table and the mattress,

$B$ is the backscatter factor,

$d_{\text {ref }}$ is the distance between the X-ray focal spot and the reference point,

$d_{\text {perp }}$ is the distance between the X-ray focal spot and the patient entrance reference point (PERP)

$f_{\text {skin }}$ is the ratio of the mass-energy-absorption coefficients from skinto-air.

It is worth noting that the correction factor for the attenuation effect of the table is actually the transmission factor.

In the definition of their calculation formula, Jones and Pasciak [30] did not include the scatter radiation from adjacent field. Neither does any of the reviewed software; however, it has a very limited influence [20].

\subsection{Output of SDC software}

As it can be seen (Table 2) outputs of SDC software can be qualitatively compared.

Almost all are color-coded according to dose level values. Use of 3D phantoms allows an easier visual interpretation of results, some can even be turned around and some are online or nearly online.

\section{Discussion}

\subsection{Comparison of software}

Table 3 details the information concerning parameters used in the SDC calculation given in formula [1] and allows the comparison between different developers.

a) CF: some developers did not specify the value used in their systems, did not define it clearly or did not use it (OpenSkin, CAATSDOSE).

However, this is of crucial importance owing to the permissive European acceptability criterion which states that the accuracy of the displayed $\mathrm{K}_{\mathrm{a}, \mathrm{r}}{ }^{1}$ shall not be higher than $35 \%$ above $100 \mathrm{mGy}$.

The CF values can be fixed (DoseWatch) or varying according to the beam energies considered (UF-RIPSA), to both acquisition and fluoroscopic imaging modes (DTS, MCAR) or to any exposure conditions [5].

In any case, currently the RDSR (36) provides the user with a single $\mathrm{CF}$. Such a single CF is probably not sufficient for an accurate calculation of skin dose, since it has to take into account the calibration of displayed $\mathrm{K}_{\mathrm{a}, \mathrm{r}}$ at different energies, which can vary. For example, heavier filtered beams $(0.1-0.2 \mathrm{~mm} \mathrm{Cu})$ exhibit a higher dependence as compared to less filtered beams. For those beam qualities the dependence is in the range of $10 \%-15 \%$ for $70-90 \mathrm{kV}$ energies [38].

b) AC: many options are possible and different ones are chosen by each provider, like data taken from the literature or a fixed value.

As a general rule, table/mattress attenuations are either based on results of physical measurements or on mathematical simulations (UFRIPSA,) for different X-ray beam projection angulations.

Attenuation values are often defined according to X-ray beam quality used for a given procedure and considering presence or absence of the table in the beam. Ideally, the beam angulation should also be considered, since it affects the path travelled by the X-rays in the table and mattress (Dose Map, DoseWatch).

From measurements on a table with a $5-\mathrm{cm}$ thick mattress, Bordier et al. [6] observed about $10 \%$ difference in the beam intensity comparing Postero-Anterior (PA) and Right Anterior Oblique (RAO) $40^{\circ}$

\footnotetext{
1 Actually, the European acceptability criterion refers to the Kerma-Area product, but a similar accuracy can be extrapolated to the Ka,r,. An identical criterion is also proposed by the IEC (2010).
}

projections. In agreement with this, DeLorenzo et al. [4] observed a decrease of $6 \%$ for RAO $40^{\circ}$ projection. It should be noted that, in addition to the mattress attenuation, Dose Map accounts explicitly for the contribution of the scatter originating from the mattress.

c) B: some variations in the choice of B values were found (see Table 2). One software used a fixed value per procedure (MCAR), whereas most software (DOSE, RDM, em.Dose, PySkinDose, OpenSkin, DIDO) used tabulated values or interpolation of those values selected according to technical parameters (possibly beam quality $(\mathrm{kV}$ and filtration), field size and phantom thickness [39]).

The most frequently used B source is clearly the simulation work of Benmakhlouf et al. $[35,36]$. In this work, B of a $15 \mathrm{~cm}$-thick water phantom were calculated for numerous beam qualities representative of modern angiography equipment with tube voltage from 50 to $150 \mathrm{kV}$ for 5 different field sizes [35]. Those Bs were later [35,36] extended to phantom thicknesses between 5 and $40 \mathrm{~cm}$. It is obvious that the choice of B has a strong influence on the accuracy of MSD calculation since the MSD is directly proportional to it, as illustrated in Table 3. Not many software use alternative approaches to estimate B.

DoseMap uses the irradiated area as unique input in a logarithmic model developed for a specific angiographic unit. Measurements on the very same $\mathrm{x}$-ray equipment showed that this approach would not cause more than $4 \%$ dose uncertainty.

Two software (DoseWatch and UF-RIPSA) refer to [32], which covers limited beam qualities compared to the extensive database of Benmakhlouf et al. [35,36]. The more complete method (FDEIR MC-GPU), based on Monte Carlo simulations, inherently takes the contribution of the scattered photons into consideration.

a) Air-to-Tissue conversion factor (f-skin): most software use a single value of 1.06 while others do not provide any information.

b) Patient model: concerning the anatomy of the patient, the simplest approaches rely on elliptical or super-elliptical models (scalable or not with real patient height and weight) or just consider the patient as a $2 \mathrm{D}$ flat surface. Other approaches use anthropomorphic phantoms voxelized from the CT images of a standard patient, such as the ICRP phantom [34] or from the digital human modelling project, or hybrid phantoms from the University of Florida - National Cancer Institute library $[40,41]$.

In order to illustrate how the use of either fixed or variable B, attenuation coefficients and patient diameters may influence SDC results, Table 3 compares two examples of cardiac procedures concerning two different average patient diameters of 20 and $35 \mathrm{~cm}$ respectively. For the whole procedure and for each patient thickness, three tube voltage ranges were considered for the assessment of the total skin dose according to equation above while $\mathrm{B}$ as well as table attenuation coefficients were alternatively considered as fixed or variable. Therefore, the same IC procedure resulted in two cumulative skin dose values, the difference of which is detailed in Table 3.

As expected, the influence of the correction of the $B$ is greater for the thicker patient, higher $\mathrm{kV}$ and heavily filtered X-ray beams, thus resulting in a dose difference between both methods reaching $20 \%$. Conversely, the influence of such a correction is significantly smaller for thinner patient, the dose difference between both methods in this case being around $6 \%$.

Knowing that an interventional cardiology procedure may result in a skin dose level of a few Gy, the consideration of physical parameters such as B and/or AC, as realistically as possible, plays an essential role. The thicker the patient, the greater the dose difference was.

According to the information presented in Table 2, each considered software may allow an accurate MSD calculation if integrating either physical, calibration measurements or input on patient's characteristics. Nevertheless, the most interesting point of the software review for the final user is the comparison of their major key features, limitations and accuracy (Table 4). This is a difficult exercise owing to the 


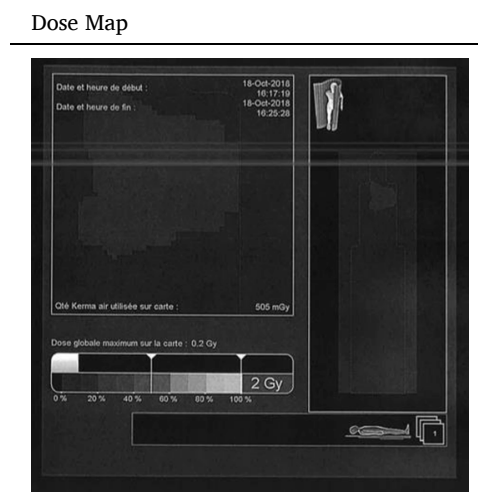

Radimetrics

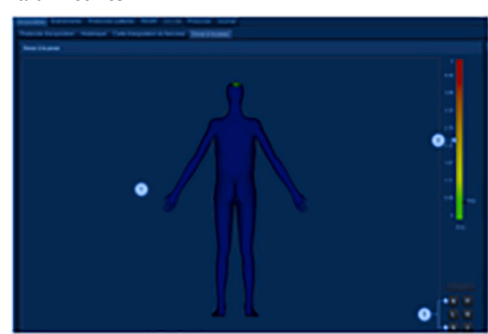

em.dose

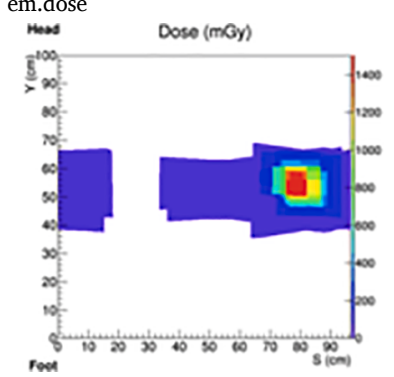

FDEIR

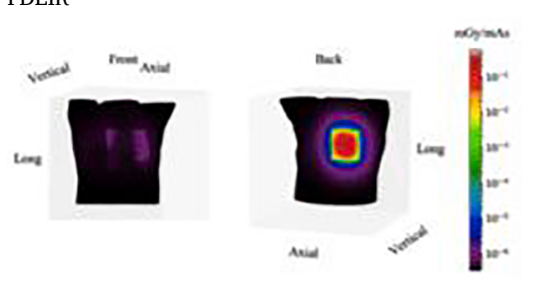

DoseWatch

DTS
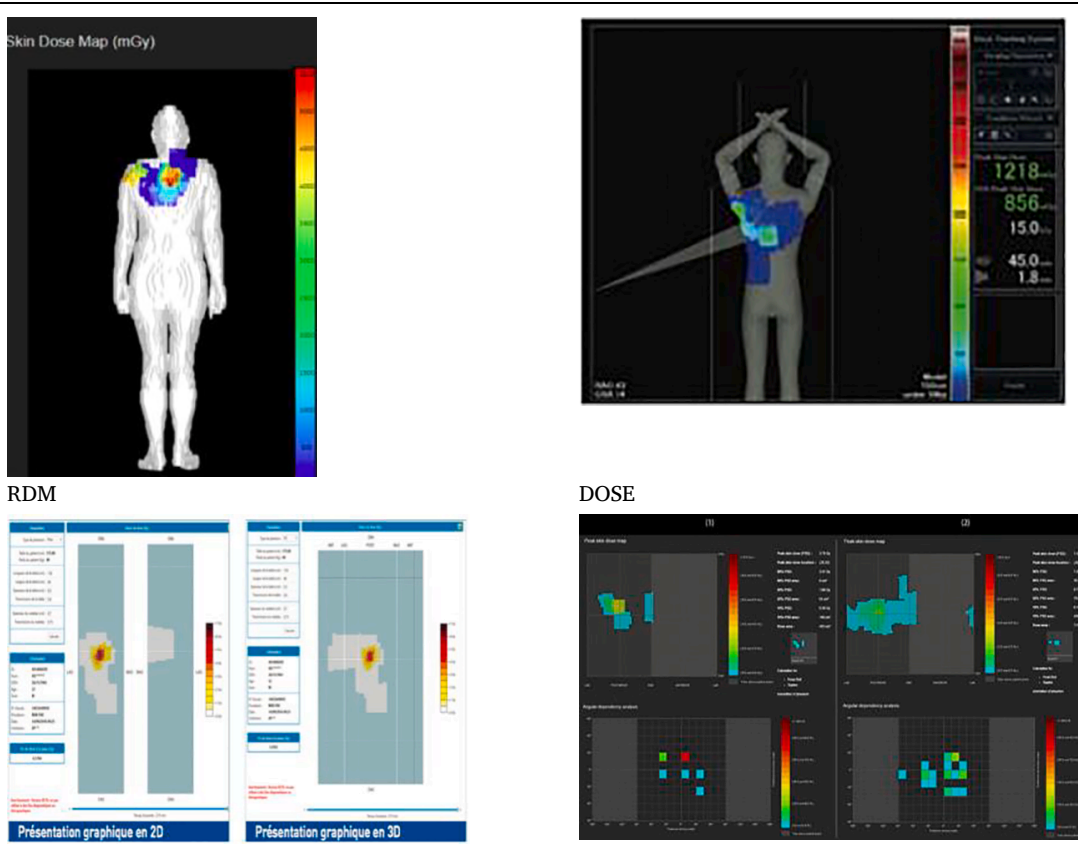

UF-RIPSA

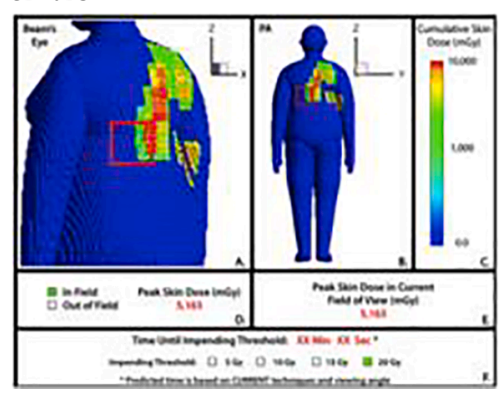

OpenSkin

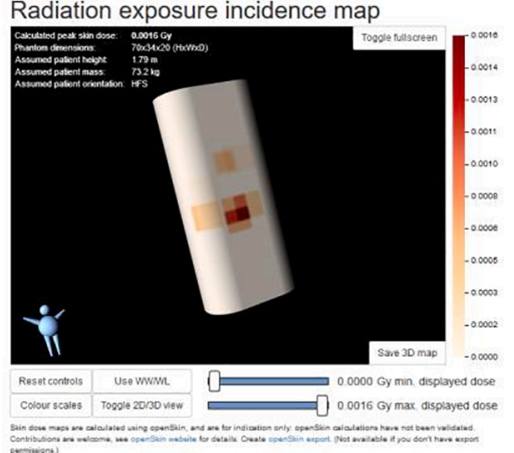

DOSE

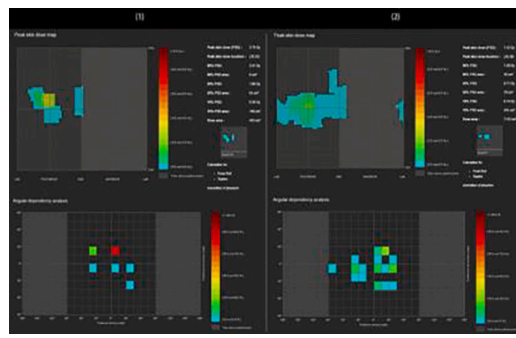

MCAR

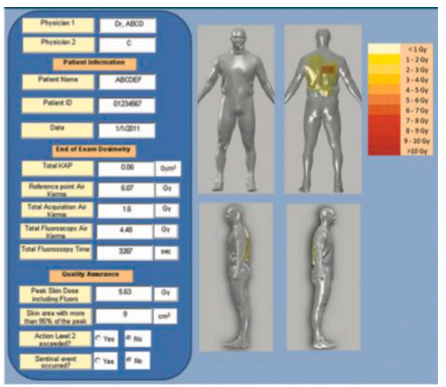

SkinCare

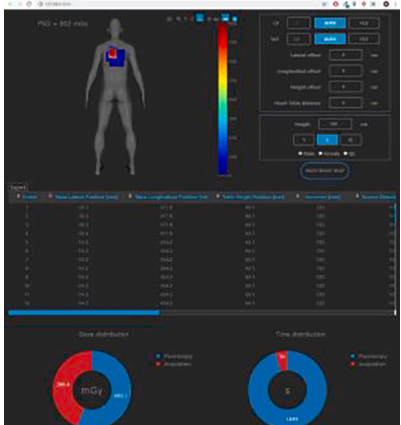




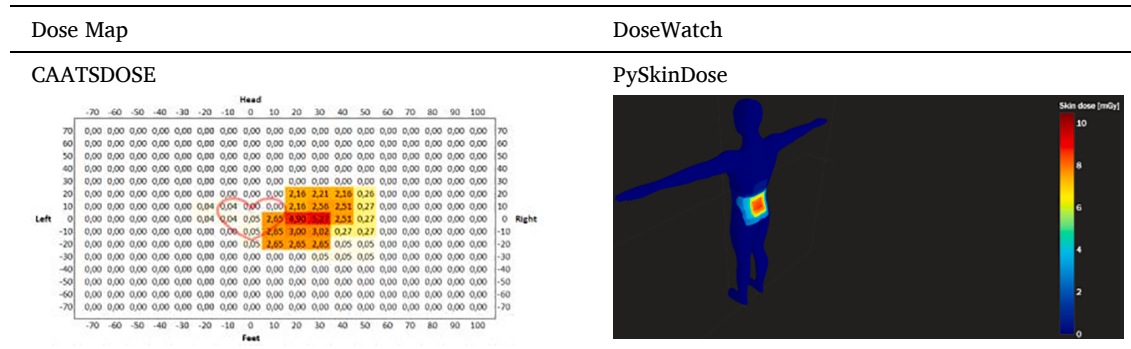

DTS

DIDO 3D
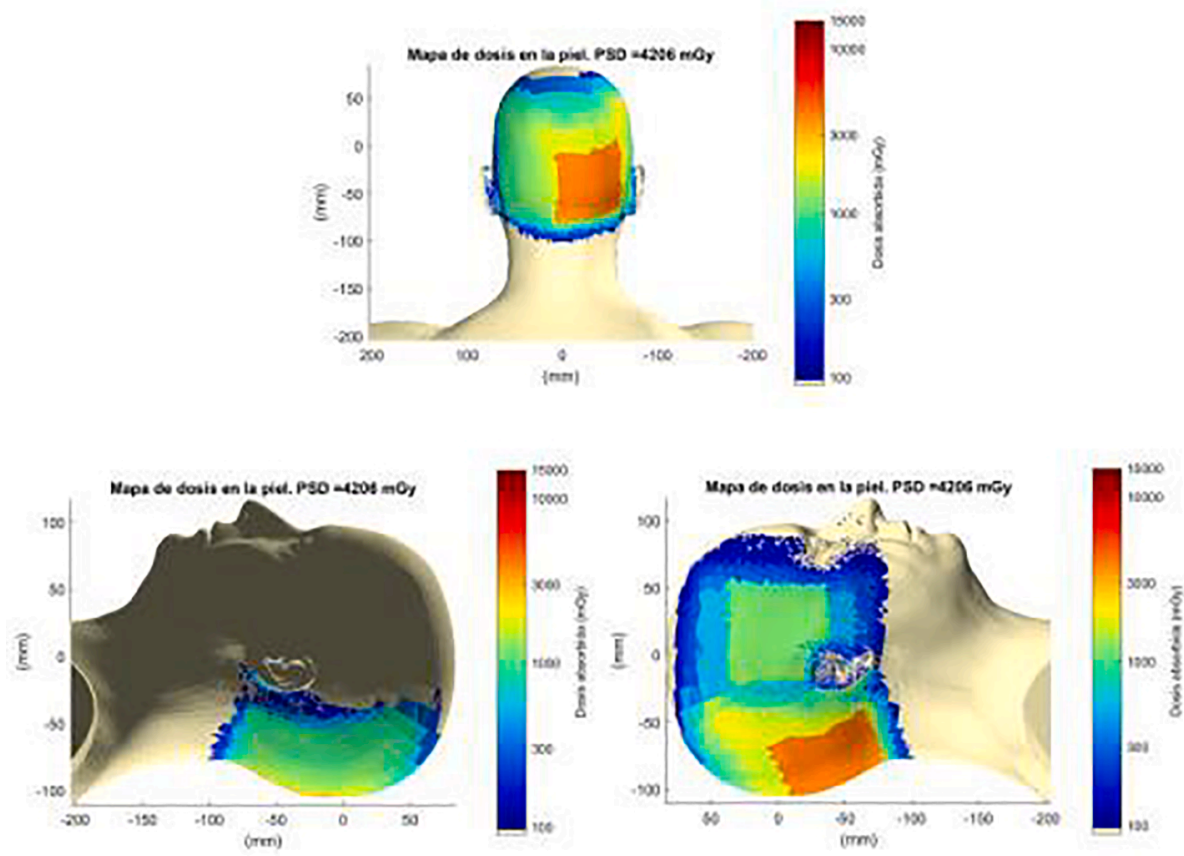

DIDO 2D

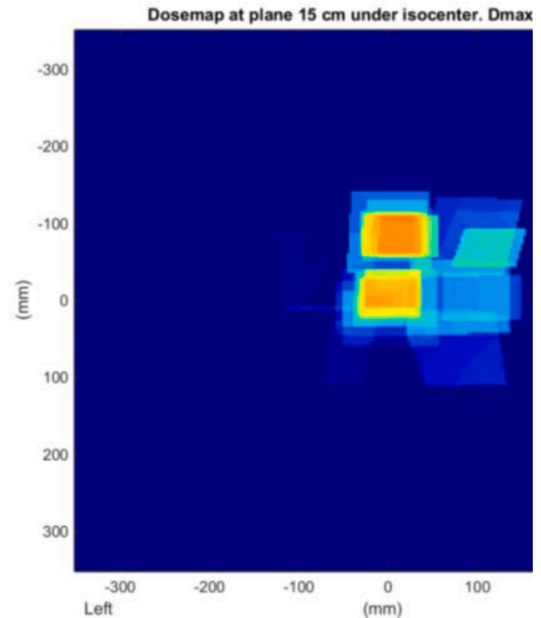


Table 3

: Comparison of CF, AC, B, f-skin and patient model by developers.

\begin{tabular}{|c|c|c|c|c|c|}
\hline $\begin{array}{l}\text { Software } \\
\text { Name }\end{array}$ & $\mathrm{K}_{\mathrm{a}, \mathrm{r}}$ calibration factor(CF) & $\begin{array}{l}\text { Attenuation coefficient of } \\
\text { table and mattress (AC) }\end{array}$ & Backscatter factor(B) & f-skin & Patient model \\
\hline Dose Map & Unknown value & $\begin{array}{l}\text { Per event, second order } \\
\text { polynomial model vs kV. }\end{array}$ & $\begin{array}{l}\text { Per event, } \\
\text { logarithmic model B } \\
=\mathrm{a} \ln (\text { area })+\mathrm{b} .\end{array}$ & Unknown value & Elliptical phantoms \\
\hline Dosewatch & 2 values per equipment & $\begin{array}{l}\text { Per event, set by user and } \\
\text { corrected for beam angulation } \\
\text { per equipment. }\end{array}$ & Per event $[32]$ & Per event [33] & Anthropomorphic phantoms [34] \\
\hline DTS & $\begin{array}{l}\text { Per event, from calibration } \\
\text { measurements. }\end{array}$ & $\begin{array}{l}\text { Per event, from calibration } \\
\text { measurements and corrected } \\
\text { for beam angulation. }\end{array}$ & $\begin{array}{l}\text { Per event, from } \\
\text { calibration } \\
\text { measurements }\end{array}$ & Single value [1.06] & CAESER database patient modelling \\
\hline em.dose & Single value set by user & $\begin{array}{l}\text { Per event, set by user for } \\
\text { different beam qualities. }\end{array}$ & Per event $[35]$ & Single value [1.06] & Elliptical phantoms \\
\hline Radimetrics & Single value set by user & $\begin{array}{l}\text { Per event, calculated, based } \\
\text { on table definition (thickness, } \\
\text { material) and Half Value } \\
\text { Layer. }\end{array}$ & Per event [35] & $\begin{array}{l}\text { Calculated based on } \\
\text { lookup tables and } \\
\text { depending on beam's and } \\
\text { Half Value Layer }\end{array}$ & $\begin{array}{l}\text { Average population phantom or if height } \\
\text { and weight or height and diameter } \\
\text { available in RDSR calculated with scaled } \\
\text { to height phantom. }\end{array}$ \\
\hline $\mathrm{RDM}$ & Single value set by user & $\begin{array}{l}\text { Per event, set by user (PA) and } \\
\text { corrected for beam } \\
\text { angulation. }\end{array}$ & Per event [35] & Per event $[34]$ & $\begin{array}{l}\text { Rectangular parallelepiped with two half- } \\
\text { cylinders on the side }\end{array}$ \\
\hline DOSE & Unknown value & $\begin{array}{l}\text { Per event, set by user and } \\
\text { corrected for beam angulation } \\
\text { and } \mathrm{kV} \text {. }\end{array}$ & Per event [35] & Unknown value & Elliptical phantoms \\
\hline UF-RIPSA & $\begin{array}{l}\text { Per event, from Kerma Area } \\
\text { Product calibration curves } \\
\text { set by user. }\end{array}$ & $\begin{array}{l}\text { Per event, from Monte Carlo } \\
\text { simulated coefficients for one } \\
\text { system. }\end{array}$ & Per event [32] & Unknown; per event & $\begin{array}{l}\text { University of Florida hybrid phantom } \\
\text { library }\end{array}$ \\
\hline MCAR & Single value set by user & Single value set by user & $\begin{array}{l}\text { Single value set by } \\
\text { user }\end{array}$ & Not implemented & $\begin{array}{l}\text { Mathematical phantoms (2011); likely } \\
\text { elliptical phantoms (2013) }\end{array}$ \\
\hline FDEIR & Unknown value & NA & NA & NA & Voxelised CT phantom \\
\hline MC-GPU & Unknown value & NA & NA & NA & Voxelised phantom \\
\hline OpenSkin & Not implemented & $\begin{array}{l}\text { From measurements on one } \\
\text { system }\end{array}$ & Per event [35] & Per event [35] & $\begin{array}{l}\text { Rectangular parallelepiped with two half- } \\
\text { cylinders on the side }\end{array}$ \\
\hline PySkinDose & $\begin{array}{l}\text { Per event, from calibration } \\
\text { measurements. }\end{array}$ & $\begin{array}{l}\text { Per event, from measurements } \\
\text { on one system. }\end{array}$ & Per event [35] & Per event [35] & No patient representation \\
\hline SkinCare & $\begin{array}{l}\text { Per event, single value set } \\
\text { by user or from } \\
\text { measurements for specific } \\
\text { system. }\end{array}$ & $\begin{array}{l}\text { Per event, single value set by } \\
\text { user or from measurements } \\
\text { for specific system. }\end{array}$ & Per event [35] & Per event [35] & $\begin{array}{l}\text { 3D human models with different sizes, } \\
\text { heights and gender }\end{array}$ \\
\hline CAATSDOSE & $\begin{array}{l}\text { From calibration Quality } \\
\text { Control measurements }\end{array}$ & Single value set by user & Per event $[35,36]$ & Single value $[1.06]$ & Flat surface \\
\hline DIDO & $\begin{array}{l}\text { From calibration at } \\
\text { standard conditions }(80 \mathrm{kV} \text {, } \\
\text { low filtration) }\end{array}$ & $\begin{array}{l}\text { Per event, (kV, filtration, } \\
\text { angle) Measured in Canon } \\
\text { Infinix } 8000 .\end{array}$ & Per event & Single value $[1.06]$ & $\begin{array}{l}\text { - plane- elliptic and anthropomorphic } \\
\text { phantoms }\end{array}$ \\
\hline
\end{tabular}

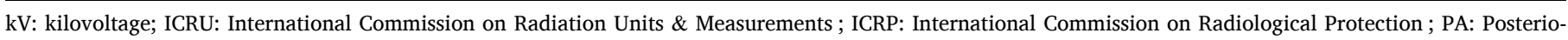
Anterior ; RDSR: Radiation Dose Structured Report; NA: Not Applicable

incompleteness of available information about the capabilities of certain software products.

Most of the software are "offline" systems and extract the data necessary to the MSD calculations from the dose report or from RDSR; only four software (Dose Map and DTS, system specific; Dose and MCGPU, streaming) provide "online" dose mapping, collecting data directly from the modality.

Unfortunately RDSR information content and position are not standardised among the vendors and even for a given manufacturer, DICOM tag can differ, thus not facilitating the extraction of the data.

Obviously, from a radiation protection point of view, "online" systems are preferable since they allow monitoring and optimisation of the MSD during the procedure. However, collecting data from the modality is cumbersome and system-specific. While the RDSR, though not always available as streaming data during the procedure, should follow specific DICOM standards and, hence, should be accessible to all software developers [37].

It appeared that most software use ray tracing techniques to identify the irradiated area, which should allow them to account correctly for the contribution of overlapping beams to the MSD. Since ray tracing algorithms have become widely accessible thanks to the increasing availability of powerful graphics workstations in the scientific and computing communities, it is reasonable to believe that all the listed software systems use such algorithms, even if such information was not available for all.

The most frequent shortcoming of the reviewed systems is surely the lack of clarity on the CF and AC used for the MSD calculation: sometimes the method applied was not clearly defined and uncertainties were not available; some developers used a single $\mathrm{CF}$ without any beam quality correction; others used a CF and/or a AC measured or simulated for one single system, which limits the transportability of the system. The same comments apply to the backscatter factors.

Very few developers (DOSE, DTS, MCAR) mentioned the shape and the uniformity of the irradiation field, owing to the presence of wedge filters or the heel effect as a source of uncertainty. It is unclear whether other developers accounted for those. Since that information is not available in the RDSR, that issue was unlikely to have been considered. Furthermore, none of the reviewed software products reports the uncertainty associated with the MSD estimation.

Output data completeness and dose reporting interactivity of each system are very dependent on developer's capability or willingness to display relevant dosimetric information: MSD, skin dose map, dose map resolution, angular distribution of different parameters like $\mathrm{P}_{\mathrm{ka}}, \mathrm{K}_{\mathrm{a}, \mathrm{r}}$, number of events etc.

Graphical representation of skin dose mapping Johnson et al. [19] as well as the intrinsic map resolution is also varying from one developer to another. Images representing the mapping of skin doses for the different software are also given in Table 5 . These representations range from 
Table 4

: Comparison of SDC with fixed or variable parameters.

\begin{tabular}{|c|c|c|c|c|c|}
\hline & Parameter & Fixed & & Variable & \\
\hline Patient diameter: $20 \mathrm{~cm}$ & & & $<80 \mathrm{kV}$ & 80 to $110 \mathrm{kV}$ greater than $110 \mathrm{kV}$ & \\
\hline \multirow[t]{4}{*}{ Half Value Layer: 3.12 mm Al @ 80 kV } & $\mathrm{B}$ & 1.4 & 1.3 & 1.4 & 1.45 \\
\hline & $\mathrm{AC}$ & 0.8 & 0.75 & 0.8 & 0.9 \\
\hline & Skin Dose AC + B (Fixed) & & 7.12 (Gy) & $\%$ difference between both SDC results: 6 & \\
\hline & Skin Dose AC + B (Variable) & & 7.56 (Gy) & & \\
\hline Patient diameter: $35 \mathrm{~cm}$ & B & 1.4 & 1.5 & 1.57 & 1.6 \\
\hline \multirow[t]{3}{*}{ Half Value Layer 5.94 mm Al @ 80 kV } & $\mathrm{AC}$ & 0.8 & 0.75 & 0.8 & 0.9 \\
\hline & Skin Dose AC + B (Fixed) & & 11.03 (Gy) & $\%$ difference between both SDC results: 19 & \\
\hline & Skin Dose AC + B (Variable) & & 13.31 (Gy) & & \\
\hline
\end{tabular}

B: Backscatter factor; AC: Attenuation coefficient; SDC: Skin Dose Calculation

Table 5

: Benchmarking of SDC software.

\begin{tabular}{|c|c|c|c|c|}
\hline Software Name & Validated on & System used for validation & MSD agreement & Reference \\
\hline Dose Map & $\begin{array}{l}\text { Water and anthropomorphic phantom } \\
\text { Patient }\end{array}$ & GE Innova IGS 530 and 540 & $\begin{array}{l}(-8.6 \% ; 25 \%)(-14 \% ; 13 \%)(-53 \% ; 76 \%) \\
(<1 \%)\end{array}$ & $\begin{array}{l}{[7][8][9]} \\
{[10]}\end{array}$ \\
\hline DoseWatch & Patient & GE Innova IGS 530 & 9.5\% with em.dose $8.3 \%$ with DoseMap & [12] \\
\hline DTS & PMMA phantom & Toshiba/Canon Infinix-i Bi-plane & $\pm 10 \%$ & [42] \\
\hline \multirow[t]{3}{*}{ em.dose } & PMMA phantom & Siemens Artis ZEE & $(-36 \% ; 53 \%)$ & [16] \\
\hline & Anthropomorphic phantom & & $(-6.3 \% ; 25 \%)$ & [17] \\
\hline & Patient & & $(-43 \% ; 34 \%)$ & \\
\hline RDM & PMMA phantom & GE Innova IGS 540Siemens Artis Zee & $(-19 \% ; 21 \%)(-24 \% ; 3 \%)$ & [18] \\
\hline DOSE & Water phantom & Few Siemens Artis and Philips Allura & $7 \%$ & [43] \\
\hline UF-RIPSA & PMMA phantom & Siemens Artis ZEE & $(-14 \% ;-6.2 \%)$ & {$[20,21]$} \\
\hline MCAR & PMMA phantom & Siemens Artis ZEE & $(-9 \% ; 5.9 \%)$ & {$[22,23]$} \\
\hline FDEIR & Water phantom & GE Innova 4100-IQ & RPL dosimeters and PHITS $\pm 6 \%$ & [24] \\
\hline MC-GPU & PMMA phantom & & TLDs : $\pm 6 \%$ Penelope Code $: \pm 1 \%$ & \\
\hline PySkinDose & & Philips Allura Siemens Artis ZEE & In progress & {$[28]$} \\
\hline SkinCare & PMMA phantom Patient & Siemens Artis & $(-37 \% ; 43.9 \%)$ & [29] \\
\hline CAATSDOSE & Patient & GE Innova & $\pm 25 \%$ with DoseMap & \\
\hline DIDO & Work in progress & Canon InfiniX & 2D version: ion chamber $5 \%$. & \\
\hline
\end{tabular}

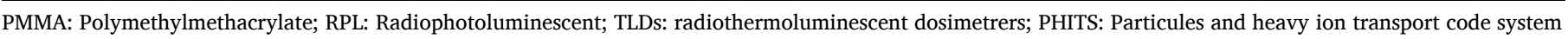

sophisticated colour codes on a 3D representation of the patient to a simple grey-scale 2D distribution on a flat representation of the patient's back, possibly including a gauge of maximum skin dose difficult to modify. This, again, underlines the lack of harmonisation of the content display, and makes an overlay of procedures performed on different systems or in different centres quite difficult. That is why the availability of a minimal set of parameters and the possibility to revert back more complex representations to a harmonized 2D mapping should be advised.

Capability of the software to be used with any kind of x-ray equipment is an essential point.

It is frequently claimed by independent, commercial software developers (em.dose, Radimetrics, RDM, DOSE, DoseWatch), while software from angiographic system manufacturers, such as DTS from Canon and Dose Map from GE, are system-specific. The same information for non-commercial software was usually not available. Open access software are able to handle RDSR of most angiographic systems at the present time.

\subsection{Comparison of software key features}

Four different classes of technical characteristics were considered in order to compare the listed SDC software:

a) the system adopted to identify irradiated area : most software used a ray tracing algorithm to identify the irradiated area (DTS, RDM, DOSE, UF-RIPSA, MCAR and Openskin)

b) skin dose map resolution : patient skin surface is mostly represented as a dense mesh the spatial resolution of which can vary according to the software developer from 0.5 to $2.5 \mathrm{~cm}^{2}$ (DoseMap, DoseWatch, RDM, Radimetrics, CAATSDOSE and MCAR)

c) potential for organ dosimetry : only a few software using Monte Carlo simulation to assess maximum skin dose may allow for other organ dose evaluation (FDEIR, MC-GPU, UF-RIPSA)

d) phantom model: several types of phantoms are used to simulate patient morphology. From a simplistic 2D representation (DoseMap, RDM, DOSE, CAATSDOSE) to a cylindrical or elliptical representation (DoseMap, Openskin, em.dose, DOSE), scalable 3D anthropomorphic phantom (DoseWatch, Radimetrics), a library 3D anthropomorphic model (DTS), a library of 3D anthropomorphic voxelized phantom (UF-RIPSA). The choice of the appropriate phantom is an important parameter regarding the MSD calculation accuracy. It is worth noting that SkinCare software, in addition to 3D human models, included a set of PMMA slabs for calibration of MSD.

\subsection{Benchmarking of SDC software}

All systems but two (Open Skin and PySkin Dose) have been validated in clinical set-ups against measurements with thermoluminescent dosimeters (TLD), optically stimulated luminescence (OSL) dosimeters, radio-photoluminescence (RPL) dosimeters or, most frequently, gafchromic films.

Most validation measurements were performed using phantoms (Polymethylmethacrylate (PMMA) slabs, water, tissue equivalent or anthropomorphic); only 3 systems (Dose Map, DoseWatch and em.dose) were validated on patients.

For phantom measurements, the calculated MSD agreed with the measurements within $\pm 25 \%$. For patient measurements, the agreement was poorer or similar to the phantom values and the MSD agreed with 
the measurements within $\pm 76 \%$ for Dose Map, within $\pm 43 \%$ for em. dose and within $\pm 25 \%$ in a more recent publication. SkinCare software results agreed within $\pm 44 \%$. It should be noted that MC-GPU and FDEIR were only validated for limited beam projections (PA and RAO $30^{\circ}$, respectively), whereas some systems were validated for several projections or sequences of projections.

Few systems were also validated against MC simulations (FDEIR, MC-GPU, UF-RIPSA) and agreed within $\pm 6 \%$. DoseWatch was compared against Dose Map and em.dose during real procedures and showed agreement within $10 \%$ on average.

It is worth noting that no software was actually physically validated on angiographic units from all vendors, though several software developers claimed vendor-independent transportability.

Software were usually validated on one, maximum two, types of angiographic units.

These wide discrepancies in the validation methods highlight the need for extended physical validation of the software.

\subsection{Limitation of SDC software}

A variety of limitations were found among the reviewed SDC software:

a) Parameter Accuracy : UF-RIPSA software specifically provides a family of AC simulated trough Monte Carlo calculation as well as CF but only for one system.

DTS calibration procedure is vendor-related, making its assessment by the local medical physicist difficult. The RDM software also makes MSD calculations using single $\mathrm{CF}$ and AC values without any correction for the beam quality while this parameter plays an important role on dose calculation accuracy (see table 3).

In many cases the way $\mathrm{CF}$ and/or $\mathrm{AC}$ are determined and taken into account in MSD assessment is:

- unknown (FDEIR, MC-GPU, OpenSkin)

- unclear (DoseMap, DOSE)

- kept constant for all beam projections (DoseWatch, RDM, UF-RIPSA, OpenSkin, PySkinDose, CAATSDOSE)

b) Software Portability : some packages (DoseMap, DTS) are vendorspecific which restricts their use to a larger scale of existing equipment. Many are not compatible with all four major vendors (Radimetrics, RDM, em.dose, DoseWatch, DIDO...). DIDO also has to be used with DOLQA dose manager system (developed in Hospital Clínico San Carlos, Madrid), but could be exported to other systems.

c) Patient Skin Representation Accuracy : A simplistic 2D patient representation is used by a few software designers (CAATSDOSE, DoseMap, em.dose, RDM, OpenSkin, MCAR) thus limiting an easy visual interpretation of MSD results. Others (DoseWatch, DTS, UF-RIPSA, MCAR, SkinCare, DIDO 3D) use a 3D representation and allow to modify shape according to patient real height and weight.

d) Results Accuracy : Reliability of MSD results is dependent on patient's position on the table. The position of the patient is not standardized so difficult to record.

e) Software Validation : Validation of software is not routinely or homogenously performed which makes MSD results comparison difficult (see Table 5). There is a need for a standardized benchmarking protocol in order to make the validation process accessible to all and to allow easy comparison of results.

f) Integral Dose Assessment : All software but one (CAATSDOSE) do not allow for summation of dose mapping for multiple procedures performed on the same patient.

\section{Conclusion}

This paper identified 22 dose calculation software products and reviewed key features and limitations for each of them, provided the information was available.

Among the analysed systems, only four were online, although they are considered as the most efficient for optimizing radiation protection of the patients. Currently, only two online systems are still maintained and upgraded routinely.

As for the offline systems, only 14 out of 20 could be analysed in detail due to lack of information for 6 systems. From the 14 analyses, 2 skin dose calculation used Monte-Carlo simulations and 12 used the same formula for analytical calculations. Major differences were found, both in use of factors embedded in the formula and in the modelling of the body of the patient.

Only 2 software allows for summation of dose mapping for multiple procedures performed on the same patient, while only one can do it for procedures performed on different machines.

Although some results claimed by developers were pretty accurate, large differences among vendors made it clear that work remains to be done before an accurate and reliable skin dose mapping is available for all patients.

\section{Declaration of Competing Interest}

The authors declare that they have no known competing financial interests or personal relationships that could have appeared to influence the work reported in this paper.

\section{Acknowledgments}

The work presented in this article was performed in the frame of the VERIDIC project. VERIDIC is part of the CONCERT project. This project has received funding from the Euratom research and training programme 2014-2018 under grant agreement No 662287.

Disclaimer.

This publication reflects only the author's view. Responsibility for the information and views expressed therein lies entirely with the authors. The European Commission is not responsible for any use that may be made of the information it contains.

\section{References}

[1] ICRP publication 103: The 2007 Recommendations of the International Commission on Radiological Protection. Annals of the ICRP. 2007;37:2-4.

[2] EURADOS Report 2019-02: Ciraj Bjelac O, Dabin J, Farah J, Järvinen H, Malchair F, Siiskonen T, Knežević Ž. Patient maximum skin dose in interventional procedures in radiology and cardiology: summary of WG 12 activities.

[3] https://www.irb.hr/eng/Divisions/Division-of-Materials-Chemistry/RadiationChemistry-and-Dosimetry-Laboratory/Projects/VERIDIC-Validation-andEstimation-of-Radiation-skIn-Dose-in-Interventional-Cardiology.

[4] Berry KE. Estimating Fluoroscopic Peak Skin Dose Using Manual Calculations: Health Phys 2018;114(6):649-53.

[5] DeLorenzo MC, Yang K, Li X, Liu B. Comprehensive evaluation of broad-beam transmission of patient supports from three fluoroscopy-guided interventional systems. Med. Phys. 2018;45(4):1425-32.

[6] den Boer A, de Feijter PJ, Serruys PW, Roelandt Jos RTC. Real-Time Quantification and Display of Skin Radiation During Coronary Angiography and Intervention. Circulation 2001;104(15):1779-84.

[7] Bordier C, Klausz R, Desponds L. Accuracy of a dose map method assessed in clinical and anthropomorphic phantom situations using Gafchromic films. Radiat Prot Dosimetry 2015;165(1-4):244-9.

[8] Bordier C, Klausz R, Desponds L. Patient dose map indications on interventional Xray systems and validation with Gafchromic XR-RV3 film. Radiat Prot Dosimetry 2015;163(3):306-18.

[9] Nilsson Althén J, Sandborg M. Verification of indicated skin entrance air kerma for Cardiac X-Ray-guided Intervention Using Gafchromic film. Radiat Prot Dosimetry 2016;169(1-4):245-8.

[10] Didier R, Bourhis D, Oueslati C, Nasr B, Le Ven F, Noël A, Jobic Y, Damien P, PeneBaverez D, Mansourati J, Nicol PP, Gilard M. In vivo validation of Dosemap software use in interventional cardiology with dosimetrics indicators and peak skin dose evaluation. Catheter Cardiovasc Interv 2019;94(2):216-22.

[11] Gardavaud F, Tavolaro S, Grussenmeyer-Mary N, Cornelis F, Boudghène F. Peak Skin Dose evaluation for vascular clinical procedures in interventional radiology: a comparison between three computation numerical solutions. Phys Med. 2018;56: 32.

[12] Greffier J, Grussenmeyer-Mary N, Larbi A, Goupil J, Cayla G, Ledermann B, Beregi JP, Frandon J. Experimental evaluation of a radiation dose management 
system-integrated 3D skin dose map by comparison with XR-RV3 Gafchromic $\mathbb{R}$ films. Physica Med 2019;66:77-87.

[13] Bednarek DR, Barbarits J, Rana VK, Nagaraja SP, Josan MS, Rudin S. Verification of the performance accuracy of a real-time skin-dose tracking system for interventional fluoroscopic procedures. In: Proceedings of SPIE-the International Society for Optical Engineering; 2011. p. 7961. 796127_1.

[14] Rana V, Rudin KS, Bednarek DR. Updates in the real-time Dose Tracking System (DTS) to improve the accuracy in calculating the radiation dose to the patients skin during fluoroscopic procedures. Proc SPIE Int Soc Opt Eng. 2013:8668-86683.

[15] Rana VK, Rudin S, Bednarek DR. A tracking system to calculate patient skin dose in real-time during neurointerventional procedures using a biplane x-ray imaging system: Biplane dose tracking system. Med. Phys. 2016;43(9):5131-44.

[16] Greffier J, Van Ngoc Ty C, Bonniaud G, Moliner G, Ledermann B, Schmutz L, Cornillet L, Cayla G, Beregi JP, Pereira F. Assessment of peak skin dose in interventional cardiology: A comparison between Gafchromic film and dosimetric software em.dose. Physica Med 2017;38:16-22.

[17] Magnier F, Poulin M, Van Ngoc Ty C, Osmond E, Bonniaud G, Coulot J, Pereira B, Boyer L, Guersen J, Cassagnes L. Comparison of Patient Skin Dose Evaluated Using Radiochromic Film and Dose Calculation Software. Cardiovasc Intervent Radiol 2018;41(5):762-71.

[18] Habib Geryes B, Hadid-Beurrier L, Waryn MJ, Jean-Pierre A, Farah J. Benchmarking the DACS-integrated Radiation Dose Monitor(R) skin dose mapping software using XR-RV3 Gafchromic(R) films. Med Phys. 2018;45:4683-92.

[19] Johnson PB, Borrego D, Balter S, Johnson K, Siragusa D, Bolch WE. Skin dose mapping for fluoroscopically guided interventions: Skin dose mapping for FGIs. Med. Phys. 2011;38(10):5490-9.

[20] Borrego D, Marshall EL, Tran T, Siragusa DA, Bolch WE. Physical validation of UFRIPSA: A rapid in-clinic peak skin dose mapping algorithm for fluoroscopically guided interventions. J Appl Clin Med Phys 2018;19(3):343-50.

[21] Borrego D, Siragusa DA, Balter S, Bolch WE. A hybrid phantom system for patient skin and organ dosimetry in fluoroscopically guided interventions. Med. Phys. 2017;44(9):4928-42.

[22] Khodadadegan Y, Zhang M, Pavlicek W, Paden RG, Chong B, Schueler BA, Fetterly KA, Langer SG, Wu T. Automatic Monitoring of Localized Skin Dose with Fluoroscopic and Interventional Procedures. J Digit Imaging 2011;24(4):626-39.

[23] Khodadadegan Y, Zhang M, Pavlicek W, Paden RG, Chong B, Huettl EA, Schueler BA, Fetterly KA, Langer SG, Wu T. Validation and Initial Clinical Use of Automatic Peak Skin Dose Localization with Fluoroscopic and Interventional Procedures. Radiology 2013;266(1):246-55.

[24] Takata T, Kotoku J, Maejima H, Kumagai S, Arai N, Kobayashi T, Shiraishi K, Yamamoto M, Kondo H, Furui S. Fast skin dose estimation system for interventional radiology. J Radiat Res. 2018;59:233-9.

[25] Badal A, Zafar F, Dong H, Badano A. A real-time radiation dose monitoring system for patients and staff during interventional fluoroscopy using a GPU-accelerated Monte Carlo simulator and an automatic 3D localization system based on a depth camera. Proc. SPIE, Medical Imaging 2013: Physics of Medical Imaging 2013; 866828. https://doi.org/10.1117/12.2008031.

[26] Rottoli F, De Mattia C, Sutto M, Colombo P, Rampoldi AG, Torresin A. Verification of skin dose values calculated by dose tracking software using GAFCHROMIC films. Phys Med. 2018;56:128-9.
[27] Colombo PE, Rottoli F, Felisi M, De Mattia C, Riga S, Sutto M, Dillion C, Massey S, Torresin A. Validation of a dose tracking software for skin dose map calculation in interventional radiology. Physica Med 2020;72:122-32.

[28] Hellström M. Estimatng patient peak skin dose with fluoroscopic procedures' department of radiation Science, Umea University - Sweden. Thesis 2018.

[29] Krajinović M, Dobrić M, Ciraj-Bjelac O. Skin dose mapping in interventional cardiology: a practical solution. Radiat Prot Dosimetry. 2020;188:1-8.

[30] Jones AK, Pasciak AS. Calculating the peak skin dose resulting from fluoroscopically guided interventions. Part I: Methods (12: 231). J Appl Clin Med Phys. 2011;15:402-3.

[31] IEC 60601-2-43. Medical Electrical Equipment-Part 2-43: Particular Requirements for the Basic Safety and Essential Performance of X-ray Equipment for Interventional Procedures. Geneva: International Electrotechnical Commission; 2010. p. 2010.

[32] ICRU Report 74. Patient Dosimetry for X Rays used in Medical Imaging 2005.

[33] ICRP Basic Anatomical and Physiological Data for Use in Radiological Protection Reference Values. ICRP Publication 89. Annals of the ICRP. 2002;32:3-4.

[34] ICRP publication 110. Adult Reference Computational Phantoms. Annals of the ICRP. 2009;39:1-166.

[35] Benmakhlouf H, Bouchard H, Fransson A, Andreo P. Backscatterer factors and mass energy-absorption coefficient ratios for diagnostic radiology dosimetry. Phys Med Biol. 2011;56:7179-204.

[36] Benmakhlouf H, Fransson A, Andreo P. Influence of phantom thickness and material on the backscatter factors for diagnostic x-ray beam dosimetry. Phys. Med. Biol. 2012;58(2):247-60.

[37] IEC 61910-1. Medical electrical equipment - radiation dose documentation - Part 1: Radiation Dose Structured Report for radiography and radioscopy. Geneva: International Electrotechnical Commission; 2015. p. 2015.

[38] Lin PJ, Schueler BA, Balter S, Strauss KJ, Wunderle KA, LaFrance MT, Kim DS, Behrman RH, Shepard SJ, Bercha IH. Accuracy and calibration of integrated radiation output indicators in diagnostic radiology: A report of the AAPM Imaging Physics Committee Task Group 190. Med Phys. 2015;42:6815-29.

[39] Sands MM, Borrego D, Maynard MR, Bahadori AA, Bolch WE. Comparison of methods for individualized astronaut organ dosimetry: Morphometry-based phantom library versus body contour autoscaling of a reference phantom. Life Sci Space Res 2017;15:23-31.

[40] Lee C, Lodwick D, Hurtado J, Pafundi D, Williams JL, Bolch WE. The UF family of reference hybrid phantoms for computational radiation dosimetry. Phys. Med. Biol. 2010;55(2):339-63.

[41] Geyer AM, O'Reilly S, Lee C, Long DJ, Bolch WE. The UF/NCI family of hybrid computational phantoms representing the current US population of male and female children, adolescents, and adults - application to CT dosimetry. Phys. Med. Biol. 2014;59(18):5225-42.

[42] Wilson SM, Prasan AM, Virdi A, Lassere M, Ison G, Ramsay DR, Weaver JC. Realtime colour pictorial radiation monitoring during coronary angiography: effect on patient peak skin and total dose during coronary angiography. EuroIntervention. 2016;12:939-47.

[43] Dedulle A, Fitousi N, Zhang G, Jacobs J, Bosmans H. Two-step validation of a Monte Carlo dosimetry framework for general radiology. Physica Med 2018;53: $72-9$. 